\title{
Coherent and ultrafast optoelectronics in III-V semiconductor compounds
}

\author{
M. Först ${ }^{*}, 1$, M. Nagel ${ }^{1}$, M. Awad ${ }^{1}$, M. Wächter ${ }^{1}$, T. Dekorsy ${ }^{2}$, and H. Kurz ${ }^{1}$ \\ ${ }^{1}$ Institut für Halbleitertechnik, RWTH Aachen University, 52074 Aachen, Germany \\ ${ }^{2}$ Universität Konstanz, Fachbereich Physik, 78457 Konstanz, Germany
}

\begin{abstract}
III-V compound semiconductors offer a fascinating multitude of phenomena which have become accessible via ultrafast time-resolved spectroscopy. Coherent vibronic and electronic dynamics are prepared by excitation with taylored femtosecond laser pulses. The analysis of their temporal dephasing or decay provides deep insights into the interaction between electronic and vibronic degrees of freedem and the surrounding bath in high purity quantum structures. In contrast to coherent electronic or vibronic states, deliberately introduced growth defects can be used to drastically shorten the lifetime of optically excited carriers. Sub-picosecond carrier lifetimes open the possibility to realize ultrafast saturable absorbers and optoelectronic transducer elements. They are particularly important as key elements in THz technology, such as efficient $\mathrm{THz}$ emitters, detectors, and for on-chip THz technology. This paper summarizes the most distinguished results relevant in the context of ultrafast optoelectronics and $\mathrm{THz}$ technology obtained in close collaboration with the Paul-Drude-Institute Berlin over the past decade.
\end{abstract}

\section{Introduction}

The celebrated microelectronic industry is rapidly approaching the limit of technical progress and of economic drive to continue unperturbedly the famous exponential development coined by Moore's law. The billion dollar silicon-based industry has been progressing along a one dimensional path driven by scaling and miniaturization of integrated circuit components.

The future evolution of microelectronics has been the most intensely debated issue since the last ten years in meetings between academia and industry. A series of valuable workshops has been held condensed in roadmap standardizations. Among them the International Technology Roadmap for Semiconductors (ITRS) played the most prominent role in both ways. ITRS can be a valuable summary to inform R\&D institutions and tool industry about the upcoming demands, but definitely slows down innovation as it is governed by the champions of low-cost manufacturing.

Taking the lessons from history of science and technology serious we should expect in these days a wave of new alternative hardware technologies taking off in the laboratories of university and research institutes that overcomes the limitations mentioned above. At the dawn of convergence of computers and telecom industry, the vast extension of photonic networks and the urgent need for parallel and ultrafast processing including new computing algorithms the future potential of advanced optoelectronics should be reconsidered carefully therefore.

\footnotetext{
" Corresponding author: e-mail: foerst@iht.rwth-aachen.de, Phone: +49 24180 27806, Fax: +49 24180627806
} 
The tragedy of III-V components in the microelectronic game up to now has been the antagony between beauty and economic success. The beauty of III-V semiconductors is the attractive compatibility of optical and electronic device technologies. Whilst the fundamental characteristics of III-V compounds and their attractive potential for band-gap engineering offer significant advantage there has been little room left where III-V technology could displace silicon as economic champion of large scale integration. Up to now the III-V family has been the material for the future and many observers believed that it will remain there although glamoured by several Nobel prizes in physics.

One of the pioneering national prestigious institutions of III-V technology has been the Paul-DrudeInstitute in Berlin directed by Klaus Ploog. Our group has benefited from the continuous progress there in MBE growth of III-V semiconductor compounds in the attempts to explore the limits of advanced optoelectronics. In this field the advent of ultrafast laser pulses opened the way to fundamental studies in time-domain of coherent vibronic and electronic properties in III-V compounds, III-V superlattices and the study of ultrafast optoelectronic transducer elements forming the basic components for the rapidly expanding field of terahertz technology. To honor the successful professorship of Klaus Ploog we would like to summarize in this article our most important results emerging from this successful collaboration with the Paul-Drude-Institute.

In the second chapter the coherent phenomena including coherent phonons and Bloch oscillations are considered and their coupling investigated in time-domain directly. In the third chapter a more application oriented issue is addressed: the use of low-temperature grown compounds as ultrafast optoelectronic switches. In the final chapter specific topics of terahertz technology are addressed, where the III-V compounds play a decisive role either on the detector or the emitter side.

\section{Coherent optoelectronics in III-V semiconductors}

The progress in the generation of ultrashort laser pulses has always been driven by the eagerness of scientists to watch physical phenomena while they happen. Like the invention of the electron microscope and scanning probe techniques have lead to atomically resolved pictures of the nanocosmos, the development of femtosecond lasers has allowed to take movies of carrier and lattice dynamics in solid state on the ultrashort time scale. From fundamental physics point of view, the sub-picosecond time scale is of special interest since carrier distributions and vibrational modes can be prepared in non-equilibrium and coherent states. In this case, ultrafast spectroscopy allows for monitoring of these phase-related excitations and the identification of involved dephasing mechanisms, relevant for a fundamental understanding non-equilibrium carrier dynamics in solid state [1].

Today, advances in molecular beam epitaxy (MBE) techniques with single atomic layer precision guarantee the provision of excellent III-V semiconductor samples. Bulk semiconductor compounds and heterostructures are therefore well-defined systems where linear properties such as energy band diagrams and phonon dispersion curves are accurately known. Beyond, band structure engineering in two-, one-, and zero-dimensional nanometer-sized semiconductor structures can preposition almost arbitrary conditions for the investigation of charge carrier and crystal lattice dynamics. Hence, III-V compounds represent ideal test systems for studies of ultrafast and coherent phenomena and attracted increasing attention over the last 20 years.

\subsection{Coherent lattice dynamics in III-V semiconductor compounds}

With the advent of picosecond pulse lasers in the 1980ies, first time resolved observations of nonequilibrium phonon dynamics in semiconductors were performed by employing so-called coherent antiStokes Raman scattering (CARS) [2]. CARS is based on time-resolving the intensity of the anti-Stokes line in a Raman scattering experiments, and hence monitors the decay times of coherently excited phonons in the time domain. This technique allowed for the first determination of decay times of longitudinal optical (LO) phonons in semiconductors on the picosecond time scale. However, this technique does 
not allow for time-resolving the coherent atomic vibrations in amplitude and phase, since in that case the laser pulse has to be shorter than half the vibrational period.

As the pulse duration is linked to the spectral width of the laser pulse via the time-bandwidth product, the bandwidth of the laser pulse necessarily exceeds the energy of the phonon. This is detrimental to CARS, since for the detection of the anti-Stokes Raman signal, the laser spectrum should not overlap with the Raman-shifted scattered light. Nevertheless, important information on the phonon decay in III-V semiconductors has been gathered by this technique [3-5]. The first time-resolved observation of coherent vibrational excitation in amplitude and phase has been reported for coherent acoustic phonons $[6,7]$. In these experiments, the interaction of the ultrashort laser pulse with the material was based on the thermoelastic effect. The absorption of the laser pulse generates a pulse of acoustic phonons, which can be traced as it propagates across a given sample structure.

The first time-resolved detection of coherent optical phonons in semiconductors was reported in the early 1990ies, almost simultanuously in the group of Ippen at MIT and in our group at RWTH Aachen University. Ippen and his co-workers observed coherent phonons in narrow-gap semiconductors and semimetals such as bismuth and antimony [8]. The coherent phonon excitation in these materials was interpreted within a model which explains the selective excitation of highly-symmetric phonon modes, the so-called displacive excitation of coherent phonons (DECP) model [9]. In our group, coherent LO phonons were observed in the bulk III-V semiconductor GaAs [10]. These optical phonon modes appear in the time-resolved reflectivity via the linear electrooptic effect due to an associated macroscopic polarization. The driving force for these excitations is the ultrafast screening of the electric surface field of the polar semiconductor on a time-scale shorter than the phonon period [11]. This model, based on the optical excitation of electron-hole pairs and their acceleration below the semiconductor surface, has often been referred to as the current surge model.

Since these first observations, coherent phonons in semiconductors have been extensively investigated. A variety of excitation mechanisms and detection techniques for the coherent crystal lattice dynamics have been identified in recent years (for reviews, see e.g. Refs. [12-14]). We will particularly show in Section 2.3 of this paper, that coherent optical phonons in a polar semiconductor can be resonantly driven by the macroscopic polarization of coherent electronic excitations.

\subsection{Coherent carrier dynamics in III-V semiconductor superlattices: Bloch oscillations}

Besides the investigation of coherent lattice dynamics in semiconductors, femtosecond spectroscopy also allows for the preparation of coherent charge carrier distributions and the observation of their ultrafast kinetics. In the coherent regime, interband excitations (e.g., coherent interband polarizations of electronhole pairs) and intraband excitations (e.g., coherent intraband polarizations of electron distributions in conduction bands) are characterized by constant phase relations between wavefunctions of the optically excited states. On the longer time scale, scattering events will destroy these phase relations and it is the goal of time-resolved spectroscopy to gain insight into the relevant coherence dephasing mechanisms.

Bloch oscillations are a prominent example of coherent intraband polarizations in semiconductor heterostructures and simultaneously represent a fundamental manifestation of the quantum theory in solid state. Their theoretical description goes back to early work of F. Bloch and C. Zener in 1928, who studied the motion of an electronic wavepacket in a periodic potential (i.e. of the crystal lattice) in the presence of a static electric field $[15,16]$. Based on a semi-classical approach, they showed that in the absence of scattering processes the wavevector $k$ of this wavepacket is uniformely accelarated across the Brillouin zone and undergoes umklapp processes at the Brillouin zone edges. In this way, the electronic wavepacket performs oscillations in real space with a frequency $v_{\mathrm{BO}}=e F d / h$, where $d$ is the lattice constant in direction of the electric field $F, e$ the electron charge and $h$ Planck's constant.

At moderate electric field strengths in a semiconductor, full cycles of Bloch oscillations can only be performed, if the lattice constant is large enough to ensure a sufficient number of transits across the Brillouin zone before scattering processes take place in reciprocal space. As proposed by Esaki and Tsu almost 40 years later, this requirement can be fulfilled in semiconductor superlattices where the lattice 
constant can be arbitrarily chosen to increase the Bloch oscillation period above typical scattering times [17]. In biased semiconductor superlattices, the picture of electronic wavepackets moving across the Brillouin zone in reciprocal space is equivalent to the coherent superposition of electronic WannierStark states $[18,19]$. Here, the frequency range in which Bloch oscillations can be observed is on the order of a few $100 \mathrm{GHz}$ to some THz. The lower limit is determined by the dephasing rate of the coherent dynamics while the upper limit is due to finite miniband widths of the superlattices.

The first experimental demonstration of coherent Bloch oscillations was facilitated by parallel advances in femtosecond laser technology and semiconductor crystal growth. In 1992, Bloch oscillations excited by ultrashort laser pulses in a biased superlattice samples were observed in four-wave-mixing experiments [20, 21]. Only one year later, the emission of electromagnetic $\mathrm{THz}$ radiation accompanied with the spatial carrier oscillations was detected in our group [22]. For a detailed overview of coherent Bloch oscillations, we refer to a recent review article by K. Leo [23]. Altough still today Bloch oscillations have only been observed upon optical excitation, the tunability of the emitted frequency in the $\mathrm{THz}$ range makes the approach of an electrically driven "Bloch oscillator" extremely attractive for advanced optoelectronic applications.

\subsection{Coupled Bloch-phonon oscillations in a biased semiconductor superlattice}

In a semiconductor, coupling phenomena between fundamental excitations have been shown to strongly modify the basic properties of the involved individual modes. Relevant for large number of optoelectronic applications, Fröhlich coupling, i.e., the interaction of electrons with optical phonons mediated by an electric field, is the dominant energy relaxation process in a polar semiconductor. Also Bloch oscillations, as a coherent excitation in a superlattice, are able to couple to fundamental excitations of the semiconductor, such as plasmons and phonons [24]. If they are tuned into resonance with the optical phonon, Bloch oscillations provide an effective driving force for coherent lattice dynamics in a semiconductor superlattice [25].

In the following, we resume the most important findings on coupled Bloch-phonon oscillations in electrically biased III-V semiconductor superlattices. The first experimental observation of Blochphonon coupling was accomplished in a GaAs/ $\mathrm{Al}_{0.3} \mathrm{Ga}_{0.7} \mathrm{As}$ superlattice, composed of $51 \AA$ thick wells and $17 \AA$ thick barriers [25]. For the excitation of coherent Bloch oscillations, a Ti:sapphire femtosecond laser was operated at a chosen wavelength to superpose Wannier-Stark states in the first electronic miniband. The superlattice was embedded in a $\mathrm{p}-\mathrm{i}-\mathrm{n}$ diode structure to enable a Bloch frequency tuning by varying the applied dc reverse bias. With a miniband width of $60 \mathrm{meV}$, the frequency of the Bloch oscillations can be tuned across the LO phonon resonance at $8.8 \mathrm{THz}$. Polarization dynamics arising from the coherently coupled Bloch-phonon dynamics were recorded in a time-resolved electrooptic detection scheme. A low excitation density of approximately $4.4 \times 10^{14}$ electron-hole pairs per $\mathrm{cm}^{3}$ and $10 \mathrm{~K}$ lattice temperature were chosen in the experiments.

Figure 1(a) depicts time-resolved electrooptic reflectivity changes for different reverse bias voltages applied to the superlattice. At positive voltages, the signals exhibit a strongly damped oscillatory contribution with increasing frequency and decreasing amplitude towards higher electric fields in the superlattice (decreasing voltages). In the middle voltage range, the signal consists of long-lived oscillations with a dephasing time on the time scale of several picoseconds. The corresponding Fourier transforms are shown in Fig. 1(b). Small and sharp peaks contribute at the GaAs TO and LO frequencies of 8.0 and 8.8 THz, respectively. Over a wide voltage range, their amplitudes do not depend on the applied voltage. Their appearance is therefore attributed to the excitation of coupled plasmon-phonon modes in highly doped cladding layers of the investigated sample [26].

In addition, spectrally broad peaks appear which show an increase of their frequency with increasing electric field strength in the superlattice. The center frequency is plotted versus the voltage applied to the superlattice in Fig. 2(a). The frequency can be identified unambiguously between 3 and 7 THz. The linear dependence on the applied voltage allows for their identification as Bloch oscillations coherently excited by the femtosecond laser pulse. For higher frequencies these peaks overlaps with the phonon 

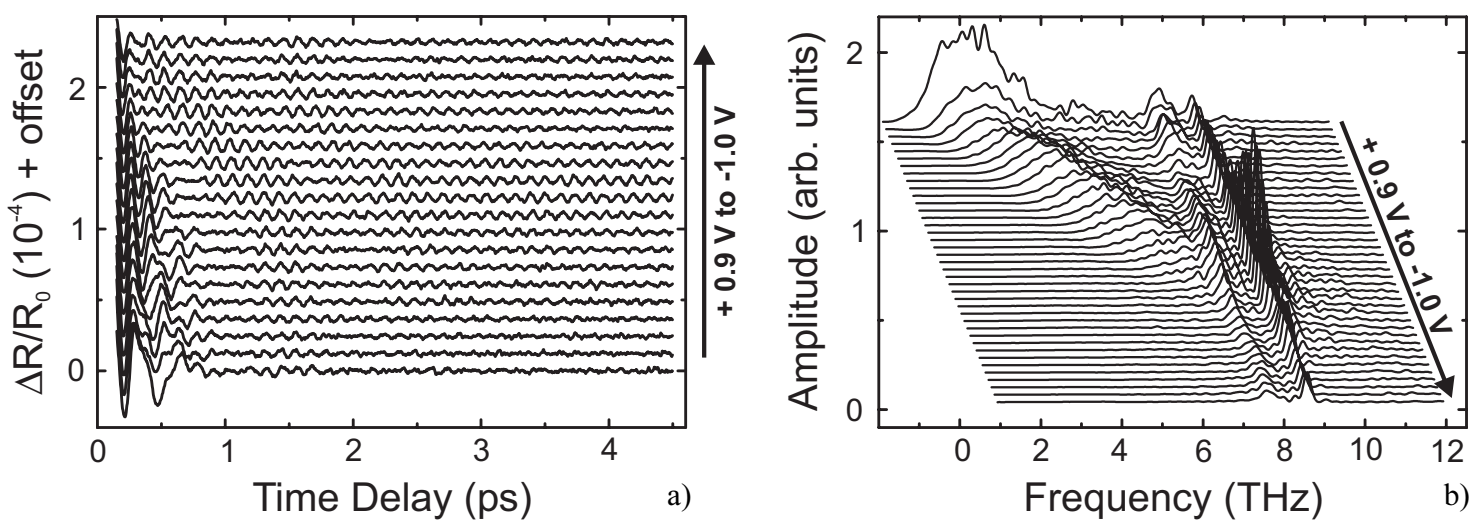

Fig. 1 (a) Time-resolved anisotropic reflectivity changes of the biased $\mathrm{GaAs} / \mathrm{Al}_{03} \mathrm{Ga}_{07} \mathrm{As}$ superlattice, detected at $4.4 \times 10^{14} \mathrm{~cm}^{-3}$ optical excitation density and $10 \mathrm{~K}$ lattice temperature. The reverse bias is changed in increments of $-0.1 \mathrm{~V}$. (b) Corresponding Fourier transforms in increments of $-0.05 \mathrm{~V}$. Figures are adapted from Ref. [25].

resonances so that a Bloch frequency cannot be assigned - although this shall not imply that Bloch oscillations are not excited.

Focussing on the voltage range in which the Bloch frequency is tuned into resonance with the LO phonon provides insight into the coupled dynamics. The most intriguing feature is the pronounced enhancement of the LO phonon amplitude for the case of resonant Bloch and LO phonon frequencies. This becomes obvious in Fig. 2(b) where the amplitude of the LO phonon (as extracted from the Fourier spectra) is plotted versus the Bloch frequency, normalized by the LO phonon frequency. Surprisingly, the absolute position of the maximum phonon amplitude deviates from the eigenfrequency the bare GaAs $\mathrm{LO}$ phonon at $8.8 \mathrm{THz}\left(v_{\mathrm{Bloch}} / v_{\mathrm{LO}}=1\right)$. Starting from the background level ascribed to the cladding layer plasmon-phonon contribution, the LO phonon amplitude passes through its absolute maximum, rapidly drops towards a minimum at the LO phonon frequency before finally passing a second local maximum. This behaviour is attributed to result from a reduced driving force for the coherent LO phonons in resonance with the Bloch oscillations. The resonant enhancement of electron-phonon scattering at the LO phonon frequency leads to increased dephasing rates of Bloch oscillations in resonance [27], and thus to a resonantly reduced driving force for the coherent optical phonons. In the theoretical treatment of Bloch-phonon coupling this effect can be considered by the introduction of a frequency dependent dephasing rate of the Bloch oscillations [24].
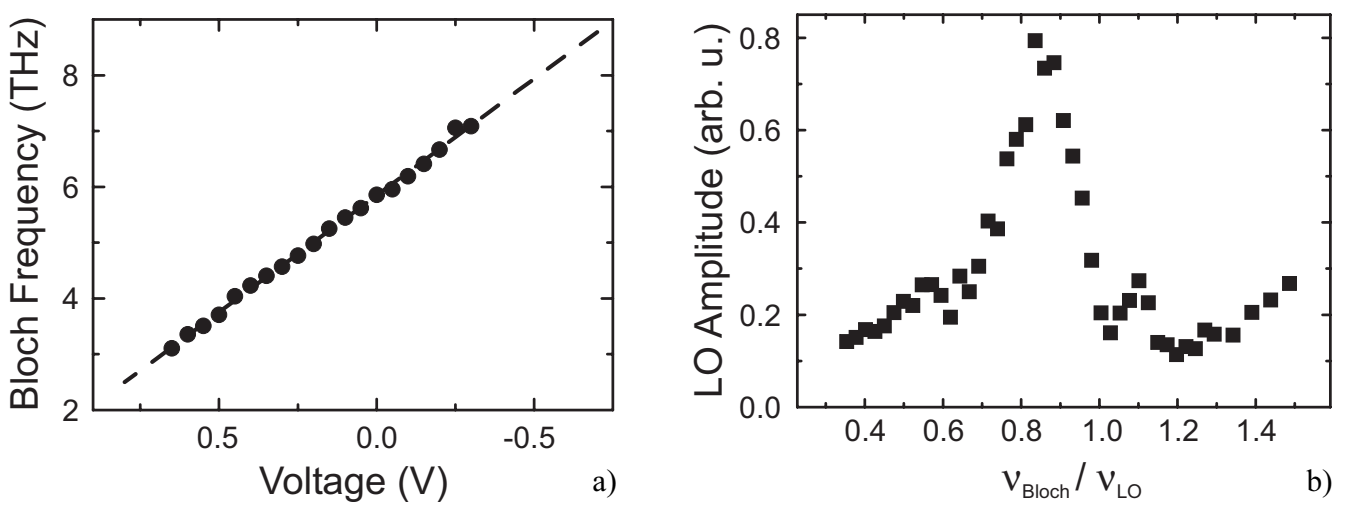

Fig. 2 (a) Frequency of Bloch oscillations as function of the voltage applied to the superlattice. The dashed line is a linear fit to the data. (b) Amplitude of coherent LO phonons versus the Bloch oscillation frequency (normalized with respect to the LO phonon frequency). Figures are adapted from Ref. [25]. 
We achieved further insight into this phenomenon in a narrow-well superlattice which was specifically designed in the $\mathrm{In}_{0.53} \mathrm{Ga}_{0.47} \mathrm{As} / \mathrm{In}_{0.52} \mathrm{Al}_{0.48} \mathrm{As}$ material system for the observation of Bloch-phonon coupling in the presence of Zener tunneling $[28,29]$. At high electric fields, Zener tunneling is an efficient dephasing mechanism of coherent Bloch oscillations in superlattices where the first electronic miniband is energetically shifted close to the confining barrier potential [30]. This dephasing process was shown to strongly modify the shape of the Bloch-phonon coupling resonance, i.e. phonon amplitudes are asymmetrically enhanced on the high frequency edge of the resonance. In addition, a selective coupling of Bloch oscillations to optical phonons of different frequencies should become feasible in the ternary system.

With regard to the observation of $\mathrm{THz}$ electromagnetic emission from Bloch oscillations [22] and coherent phonons, semiconductor superlattices biased into the Bloch-phonon resonance are expected to be strong emitters of electromagnetic radiation at the LO phonon frequency. Because of the longer dephasing time of the coherent lattice mode, room temperature emission can be obtained on time scales exceeding the lifetime of pure electronic Bloch oscillations. This effect might provide a reasonable way to obtain amplification of the $\mathrm{THz}$ emission from superlattice systems.

\section{Ultrafast carrier recombination in low-temperature grown semiconductor compounds}

The coherent dynamics presented above demonstrate how the electronic and phononic properties of a semiconductor can be manipulated and controlled in layered, epitaxially grown nanostructures. In the following section we will introduce low-temperature grown III-V semiconductor compounds, in which nanometer-sized metallic precipitates strongly reduce the lifetime of electron-hole pairs. These semiconductors with tailored carrier lifetimes are a key issue for ultrafast optoelectronic devices. In highly defect-free semiconductors like MBE-grown GaAs the carrier lifetime determined through the radiative recombination exceed values of 1 nanosecond. However, for ultrafast detectors or emitters in the $\mathrm{THz}$ frequency range this time constant would result in carrier accumulation which hence would limit the performance to the frequencies in the lower $\mathrm{GHz}$ range.

\subsection{Sub-picosecond carrier recombination in LT-grown GaAs}

A key material for ultrafast optoelectronic device is GaAs grown at reduced lattice temperature and increased As partial pressure in a molecular beam epitaxy (MBE) chamber [31]. Growth conditions in the range of $180{ }^{\circ} \mathrm{C}$ to $300{ }^{\circ} \mathrm{C}$ substrate temperature and $\mathrm{Ga}: \mathrm{As}_{4}$ equivalent beam pressures of $6: 4$ lead to the incorporation of excess As in the material. Due to the reduced growth temperature in comparison with standard GaAs MBE growth temperatures around $400-800{ }^{\circ} \mathrm{C}$ this material has been denoted as low-temperature grown GaAs (LT GaAs). The excess As in the range of $1 \%$ is mostly incorporated as As occupying a $\mathrm{Ga}$ position (neutral or positively charges $\mathrm{As}_{\mathrm{Ga}}$ antisite). Subsequent annealing of As-rich GaAs leads to the reduction of lattice imperfections with an increase in mobility and the formation of As clusters [32], however, with an $\mathrm{As}_{\mathrm{Ga}}$ antisite density still in the range of $10^{18} \mathrm{~cm}^{-3}$ [33]. The free-electron lifetime due to trapping in positively charged $\mathrm{As}_{\mathrm{Ga}}$ antisites can lie for optimized material below $1 \mathrm{ps}$ which enables the realization of devices with a frequency response in the THz frequency range [34, 35]. This lifetime is required for the realization e.g. of ultrafast $\mathrm{THz}$ emitters [36], generators for ultrafast electrical pulses [37], THz detectors or devices for down-converting $\mathrm{CW}$ light from laser diodes down to the $\mathrm{THz}$ frequency range via frequency mixing [38]. For generators of $\mathrm{THz}$ radiation the energy of the emitted radiation is provided by the static electric field in the optically excited semiconductor.

In order to achieve maximum conversion efficiency from the optical excitation to the $\mathrm{THz}$ radiation the static electric field, the carrier mobility on a sub-ps time-scale and the excited carrier density should be maximized. These requirements can be met with LT GaAs which exhibits beside the sub-ps relaxation dynamics simultaneously a higher breakdown field (up to $5 \times 10^{5} \mathrm{~V} / \mathrm{cm}$ ) in comparison to high-purity GaAs $\left(3 \times 10^{4} \mathrm{~V} / \mathrm{cm}\right)[39]$. It should be pointed out that obtaining a high-mobility LT GaAs material with 

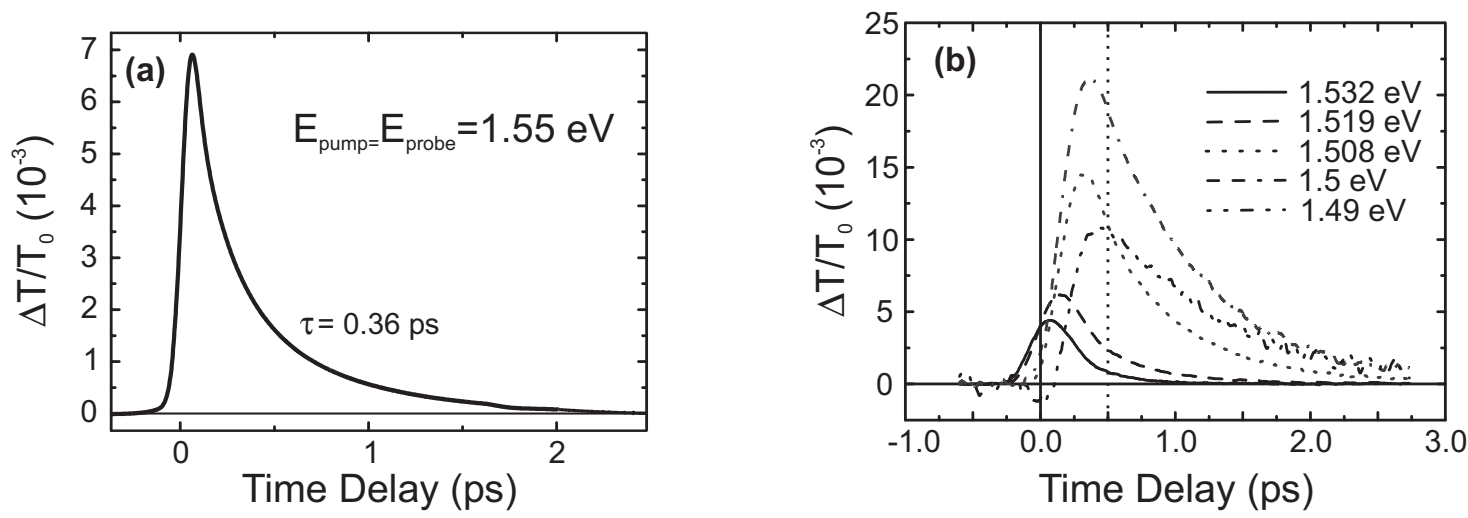

Fig. 3 (a) Time-resolved transmission changes of a $1 \mu \mathrm{m}$ thick LT GaAs film at a degenerate pump-probe energy of $1.55 \mathrm{eV}$. The optically excited electron-hole pair density is $5 \times 10^{17} \mathrm{~cm}^{-3}$. (b) Time-resolved transmission change of LT GaAs at different probe energies (adapted from Ref. [42]). The solid vertical line represents zero-time delay and the dashed vertical line indicates the delay in the maximum transmission change at the lowest probe energy.

sub-ps carrier lifetimes requires a careful adjustment of the growth and annealing parameters in a large parameter space. Alternative routes for obtaining similar material properties as those of LT GaAs are based on the growth of Er-rich GaAs [40] or implantation and subsequent annealing of GaAs with As [41].

For optimization of LT GaAs femtosecond pump-probe experiments are essential. Figure 3(a) depicts the changes of the transmission of a $1 \mu \mathrm{m}$ thick LT GaAs layer recorded in a femtosecond degenerate pump-probe experiment at $1.55 \mathrm{eV}$ at $8 \mathrm{~K}$ lattice temperature. After the increase in transmission due to bleaching of interband transitions by the pump pulse the signal decays with a mono-exponentional decay constant of $360 \mathrm{fs}$. The transmission returns to its unperturbed value at $1.5 \mathrm{ps}$ indicative of a complete relaxation of carriers from the conduction band via non-radiative relaxation processes into defect states. For a complete picture of the relaxation process including interband relaxation via Fröhlich interaction and the trapping process into defect states we performed spectrally resolved pump-probe experiments close to the band-edge [42] and two colour pump-probe experiment with sub-bandgap probe energies obtained from a parametric-optical oscillator (OPO).

Figure 3(b) shows the transmission changes recorded of a LT GaAs film at different probe energies close to the bandgap. Depending on the probe energy, the maximum of the transmission change is shifted to longer time delays. This behaviour indicates a relaxation of free carriers in the conduction band into localized states close below the unperturbed band gap and a subsequent relaxation into deep traps with a one ps time constant [42]. Recombination of carriers within the deep levels is accessible by two-colour pump-probe experiments with sub-bandgap probe energies provided by an OPO [43]. Figure 4 shows the absorption changes recorded at $0.85 \mathrm{eV}$ on a logarithmic scale. The positive change in absorption change in contrast to the negative one in the degenerate pump-probe above the bandgap is based on free carrier absorption and carrier reabsorption from midgap states. Hence the filling and recombination of the midgap traps becomes accessible. The longer time constant of $10 \mathrm{ps,} \mathrm{which} \mathrm{was} \mathrm{absent} \mathrm{in} \mathrm{the} \mathrm{above-}$ bandgap pump-probe is a clear indication of the recombination dynamics. The optical excitation density of $10^{17} \mathrm{~cm}^{-3}$ is well below the density of the midgap traps, hence no saturation effects are observed. Experiments at higher pump-excitation levels allow obtaining the density of the midgap traps from the saturation behaviour [44, 45].

\subsection{LT-grown InGaAs/GaAs multiple quantum wells}

The unique optical and electrical properties of low-temperature grown GaAs associated with the ultrashort free carrier lifetimes and high electron mobilities has opened way for its application in a variety of optoelectronic devices (see Section 4). However, the fundamental bandgap of LT GaAs (about 

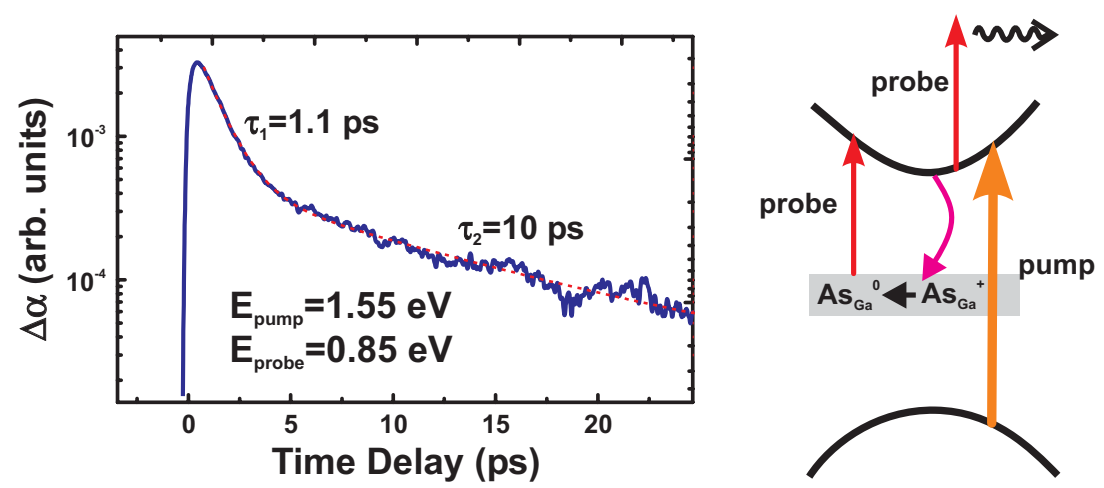

Fig. 4 (online colour at: www.pss-b.com) Left: Time-resolved absorption change of a $1 \mu \mathrm{m}$ thick LT GaAs film obtained with a two-colour pump-probe experiment on a logarithmic scale at room temperature (blue line). The pump energy is $1.55 \mathrm{eV}$ and the probe energy is $0.85 \mathrm{eV}$. The red dashed line is a double exponential fit to the experimental data with time constants of $1.1 \mathrm{ps}$ and $10 \mathrm{ps}$, respectively. Right: Schematic sketch of the free-carrier absorption and the absorption from mid-gap states at $0.85 \mathrm{eV}$.

$1.42 \mathrm{eV}$ at room temperature) requires the use of laser sources operating at wavelengths longer $870 \mathrm{~nm}$. Since the interest in applications at infrared telecommunication wavelengths is steadily growing, a semiconductor material, opaque at these wavelengths and of comparably favoured properties, is highly desired. III-V compounds of the ternary $\operatorname{In}_{x} \mathrm{Ga}_{1-x}$ As system might be the material of choice. Their electronic bandgap can be tuned over a broad wavelength range by variations of the indium and gallium element concentrations as well as additional contributions of strain-induced bandgap shifts, if these compounds are grown lattice-mismatched on GaAs or InP substrates.

The following study demonstrates that free carrier lifetimes in the picosecond range can also be realized in LT-grown $\operatorname{In}_{x} \mathrm{Ga}_{1-x} \mathrm{As}$ compounds. To this end, we analyzed the ultrafast response of optically excited charge carriers in LT-grown strained InGaAs/GaAs multiple quantum wells (MQWs). The investigated structures - fabricated at the Paul-Drude-Institute - consist of 50 periods of $6.2 \mathrm{~nm}$ thick $\mathrm{In}_{0.25} \mathrm{Ga}_{0.75} \mathrm{As}$ wells $6.0 \mathrm{~nm}$ thick GaAs barriers deposited on a GaAs substrate. The samples were grown by molecular beam epitaxy at different substrate temperatures between 200 and $380^{\circ} \mathrm{C}$ and subsequently annealed at $620^{\circ} \mathrm{C}$ for 15 minutes. The bandgap of the specific samples was designed for their implementation as ultrafast saturable absorbers for passive modelocking in pulsed lasers around $1.06 \mu \mathrm{m}$. However, changing the quantum well thickness and $\operatorname{In}_{x} \mathrm{Ga}_{1-x}$ As composition enables a shift of the electronic bandgap to near infrared telecommunication wavelengths.

Figure 5(a) shows static absorption spectra of these InGaAs/GaAs MQWs grown at $200^{\circ} \mathrm{C}$ and $380^{\circ} \mathrm{C}$. A distinct excitonic resonance at $1025 \mathrm{~nm}$ is only observed in the structure grown at the elevated temperature, while the band edge is significantly smeared out in the InGaAs MQW grown at $200{ }^{\circ} \mathrm{C}$. The disappearance of the excitonic feature may result from large inhomogeneities of the band-to-band transition energies associated with potential fluctuations, internal field inhomogenities, or short carrier lifetimes which arise from the formation of As precipitates during the growth and annealing process [42].

Ultrafast carrier dynamics in the LT-grown InGaAs/GaAs quantum well samples were investigated in pump-probe experiments at room temperature. The experiments were carried out with laser pulses of $120 \mathrm{fs}$ duration and $13 \mathrm{~nm}$ spectral width wavelength-tunable close to the electronic bandgap of the investigated quantum wells.

Figure 5(b) shows time-resolved transmission changes of the $200{ }^{\circ} \mathrm{C}$ grown sample traced at different wavelengths between $1010 \mathrm{~nm}$ and $1040 \mathrm{~nm}$. For all excitation wavelengths a tiny dip is observed at zero time delay which is ascribed to a two-photon absorption contribution from the GaAs substrate. Subsequently, the transmitted intensity increases on the time scale of the laser pulse duration. The optical excitation of carriers from the valence to the conduction band bleaches the electronic transition predominantly by phase state filling and the transmission of the MQW structure increases [46]. As for the case of 

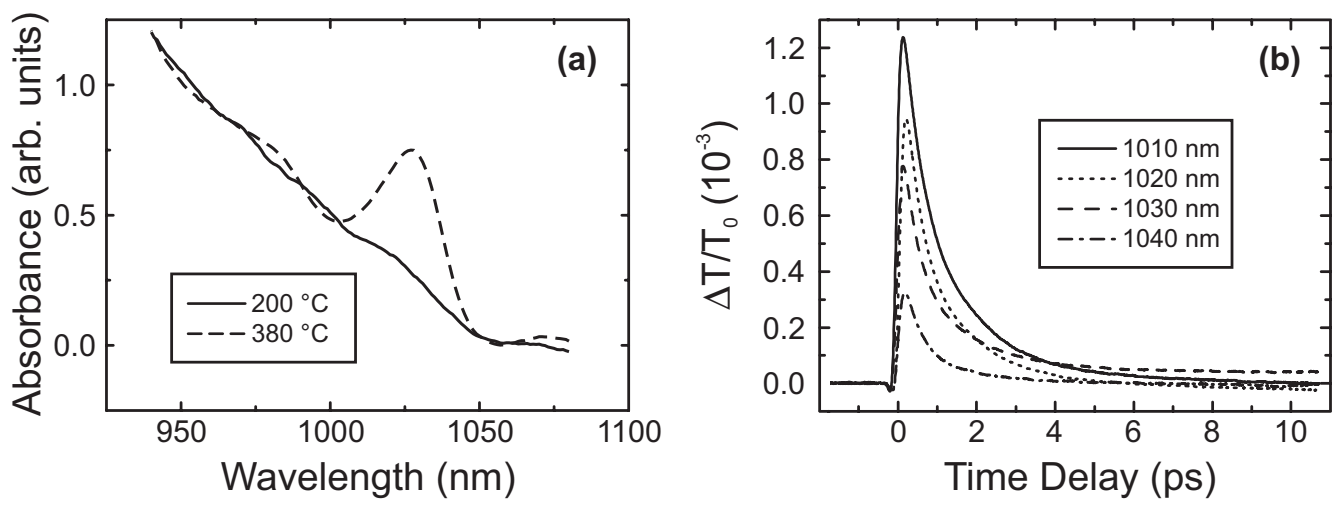

Fig. 5 (a) Room temperature absorption spectra of strained InGaAs/GaAs MQWs grown at 200 and $380^{\circ} \mathrm{C}$ substrate temperature. (b) Time resolved transmission changes of the MQW sample grown at $200{ }^{\circ} \mathrm{C}$. The excitation wavelength is varied in the vicinity of the electronic bandgap.

LT GaAs, the amplitude of the nonlinear transmission decays at positive time delays as a result of relaxation and recombination dynamics of photogenerated electron hole pairs. The amplitude of the peak transmission at zero time delay increases with shorter wavelengths due to increased light absorption above the bandgap (see Fig. 5(a)). This is in notable contrast to the MQW sample grown at $380{ }^{\circ} \mathrm{C}$ where the peak transmission exhibits a clear maximum at the excitonic resonance wavelength around $1025 \mathrm{~nm}$ (not shown here).

The relaxation and recombination dynamics can be quantified by double-exponentially fitting the temporal decay of the nonlinear MQW transmission. From this analysis, slow and fast components to the nonlinear optical response are identified for each sample. A short time constant below $350 \mathrm{fs}$ is obtained for all samples, and is therefore assigned to fast carrier relaxation. On the other hand, the time constant of the slow decay component associated with the carrier recombination process strongly depends on the sample growth temperature. For a direct quantitative comparison between different samples, these time constants are determined from nonlinear transmission changes above the bandgap, i.e. at $1010 \mathrm{~nm}$ wavelength. Figure 6 depicts decay times obtained for different sample growth temperatures. The free carrier lifetime decreases with lower growth temperatures and reaches a minimum of about $1.5 \mathrm{ps}$ for the MQW sample grown at $200{ }^{\circ} \mathrm{C}$.

The clear dependence of the free carrier lifetime on the growth temperature indicates, that in analogy to LT-GaAs the incorporation of excess As during growth and the formation of precipitates during subsequent annealing play a significant role. In addition, contributions from strain relaxation accompanied with dislocation generation at As precipitates during the annealing process of LT-grown strained InGaAs/GaAs MQWs might be taken into account [47]. An electron transmission spectroscopy study of

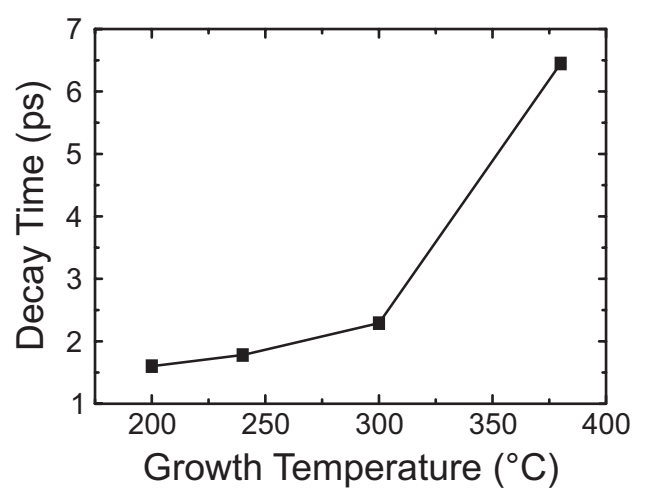

Fig. 6 Free carrier lifetime of InGaAs/GaAs MQW samples grown at temperatures between 200 and $400{ }^{\circ} \mathrm{C}$, as determined from double-exponential fits to time-resolved transmission changes above the band edge. 
the samples investigated here can provide further insight which is beyond the scope of this study. A further reduction of the free carrier lifetime in ternary $\operatorname{In}_{x} \mathrm{Ga}_{1-x} \mathrm{As}$ compound system below 1.5 ps seems feasible by a thorough optimization of the growth and annealing parameters.

\section{Application of LT-GaAs photoconductive switches in THz devices}

The extremely short free carrier lifetimes of low-temperature grown semiconductor layers offer significant advantages for the design of emitters and detectors in the $\mathrm{THz}$ frequency range. During the development of integrated $\mathrm{THz}$ systems, a lot of effort has to be directed towards the reduction of signal loss. In comparison to the microwave range, losses of waveguide-based integrated systems are unequally higher since metallic, dielectric and radiation losses usually all increase as frequency increases into the $\mathrm{THz}$ range. Current approaches for low-loss $\mathrm{THz}$ waveguides include for example polymer- or air-filled metallic $[48,49]$ or dielectric waveguides $[50,51]$. As described in the following sections, a very attractive way to realize integrated devices based on these waveguide technology platforms, utilizing the superb properties of LT-GaAs for THz Signal excitation and detection, is given by the epitaxial lift-off technique [52], which enables the bonding of thin layers of semiconductor material to arbitrary substrates. The epitaxial lift-off technique saves PC material and offers a high degree of freedom in the choice of substrates, e.g., to use optically transparent substrates for backside excitation or to reduce the dielectric load of a probe-tip by choosing thin low-k films as a substrate.

\subsection{Epitaxial lift-off fabrication techniques}

The fabrication of waveguide-based $\mathrm{THz}$ devices with integrated photoconductive (PC) LT-GaAs switches starts with the fabrication of LT-GaAs material. The wafers used for our studies are fabricated at the Paul-Drude-Institute in Berlin by molecular beam epitaxy (MBE). A lattice-matched layer system consisting of $150 \mathrm{~nm}$ GaAs, $100 \mathrm{~nm}$ AlAs and $1300 \mathrm{~nm}$ LT-GaAs is deposited on a 100 oriented SI-GaAs wafer. The deposition temperatures of AlAs and LT-GaAs are 550 and $200{ }^{\circ} \mathrm{C}$, respectively. The wafers are finally annealed at $615^{\circ} \mathrm{C}$ for $15 \mathrm{~min}$. The carrier lifetime of our LT-GaAs samples measured by reflection spectroscopy is typically in the order of $180 \mathrm{fs}$.

The final devices are made by transferring laterally structured pieces ("chips") of the LT-GaAs layer to a host substrate (HS). LT-GaAs chips are defined as needed by first depositing and structuring a photo-resist layer on top of the LT-GaAs material. In the next step the LT-GaAs is etched by chemical wet etching in a solution of $\mathrm{H}_{2} \mathrm{O}_{2}: \mathrm{H}_{2} \mathrm{SO}_{4}: \mathrm{H}_{2} \mathrm{O}$ (volume ratio $8: 1: 1$ ). The LT-GaAs chips are then lifted from the GaAs wafer by highly selective wet-etching of the AlAs sacrificial layer underneath the LT-GaAs in $10 \%$ HF solution for a few hours.

Three different concepts as sketched in Fig. 7 have been used for the fabrication of integrated PC switches on host substrates. The first two concepts differ by the order of the process steps of LT-GaAs transfer and metal electrode (planar waveguide) structuring on the HS [53, 55]. In a third alternative, the electrode structures are defined on the LT-GaAs chip before it is transferred to the HS. While the first

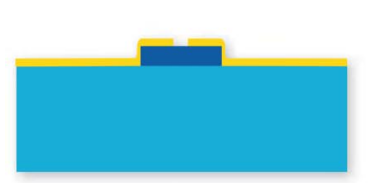

(a)

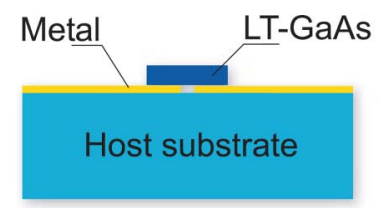

(b)

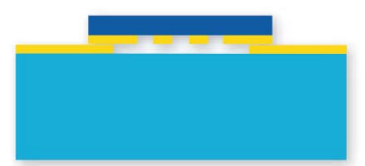

(c)

Fig. 7 (online colour at: www.pss-b.com) Schematic plots of the three different concepts applied for the fabrication of integrated PC switches: (a) LT-GaAs thin-film bonding to host substrate (HS) first, (b) metal deposition and structuring on the HS first and (c) metal deposition an structuring on LT-GaAs before transfer to the HS. 
two methods are preferably used to deposit small LT-GaAs chips down to $30 \mu \mathrm{m} \times 30 \mu \mathrm{m}$ in size with relatively large separation onto the HS, the last method is used to transfer larger pieces of LT-GaAs of up to some square millimeters area with closely spaced electrodes [54]. In all cases, the mechanism responsible for adhesion is van-der-Waals bonding. The bonding force depends on the HS material and its surface-roughness. LT-GaAs adhesion sufficiently strong to conduct post processing as required in the concept of Fig. 7(a) can be observed on a multitude of polymeric substrates. Substrates with lower bonding force capability may require the concept depicted in Fig. 7(b). However, in this case, it is even more important to avoid particles between the LT-GaAs and the HS in order to achieve a reliable electrical contact to the LT-GaAs.

\subsection{Application examples: THz waveguide, probe-tip and antenna devices}

The first example of bonded LT-GaAs chips used for integrated PC switches we want to discuss, is focused on the excitation of THz pulses on thin-film microstriplines (TFMSLs) with the low-k polymer benzocylobutene (BCB) as dielectric [55]. As sketched in Fig. 8(a) a TFMSL consists of a ground metal layer on a substrate, covered by a dielectric layer with a metallic strip line on top. Such TFMSLs may be regarded as the terahertz counterpart to conventional larger microstriplines used in microwave integrated circuits. They are now increasingly used as sensing elements for biomolecule detection at THz frequencies [56-59]. For TFMSLs, BCB is a very popular dielectric material, since in addition to having a relatively low dielectric loss tangent, it provides also chemical robustness. Hence, BCB-based TFMSLs have been used at cryogenic temperatures [60] and in various fluid environments [57, 59]. Another attractive property of $\mathrm{BCB}$ is its applicability as transducer material to optically probe the electric field of a $\mathrm{THz}$ signal transmitted through a TFMSL by electric field induced second-harmonic (EFISH) generation [61].

Figure 8 displays the configuration considered here with a LT-GaAs PC switch integrated into a TFMSL using the method of Fig. 7(a). The $3 \mu \mathrm{m}$ wide stripline constists of a Ti/Au layer with a thickness of $700 \mathrm{~nm}$. The BCB dielectric layer has a thickness of $12 \mu \mathrm{m}$. Further details of fabrication have been described in [61]. For time-domain EFISH measurements a mode-locked Ti:sapphire laser emitting $100 \mathrm{fs}$ optical pulses at $805 \mathrm{~nm}$ wavelength with a repetition rate of $75.6 \mathrm{MHz}$ is applied. The setup is based on a pump probe scheme, where pump pulses with $10 \mathrm{~mW}$ average power excite the $20 \mathrm{~V}$ biased PC gap in the TFMSL. Temporally delayed probe pulses with $45 \mathrm{~mW}$ average power sample the electric $\mathrm{THz}$ signal transients within the polymer. The probe beam is focused on the device to a spot diameter of $10 \mu \mathrm{m}$.

In the SHG experiments we detect the p-polarized second-harmonic response $I_{\mathrm{pp}}^{2 \omega}$ generated by a p-polarized fundamental field $E_{\mathrm{p}}^{\omega}$, where p denotes the direction parallel to the plane of incidence. Timeresolved measurements are performed with the plane of incidence vertical to the signal conductor, as shown in Fig. 8(a). In this configuration the THz field and the optical probe field have the same polarization and a maximum signal response is obtained. Generation of the probe signal is based on third-order nonlinear polarization $P^{3}(\omega, \omega, 0)$ in the polymer giving rise to a quasi-dc electric field induced secondharmonic optical signal. Here, the probe signal can be obtained in the BCB material as it is. Unlike second-order nonlinear polymers it requires no additional processing like e.g. poling. In comparison to other on-chip probing schemes using external probe-tips [62] this internal field probe is highly non-invasive. Since no dielectric load is introduced to the device, the $\mathrm{THz}$ signal can be sampled with ultra-high bandwidth without generating parasitic distortions. Figure 8(b) shows a THz pulse sampled at a short distance to the LT-GaAs switch on the TFMSL. Pulse width and rise time are only $410 \mathrm{fs}$ (FWHM) and $300 \mathrm{fs}$, respectively. The corresponding FFT signal reveals a bandwidth of ca. $3 \mathrm{THz}$, which is to the best of our knowledge the highest bandwidth demonstrated for an on-chip transmitted electric signal.

However, in some cases it may not be possible to use the dielectric material of the device for nonlinear optical electric field probing, for example if the optical absorption is too large or nonlinear optical tensor elements are too small to produce an adequate field-induced signal. In that case, the integration of further PC switches for signal detection as well as for generation can be a viable alternative solution. This approach was applied to $\mathrm{THz}$ bio-chip sensor arrays with $\mathrm{PC}$ on-chip $\mathrm{THz}$ pulse generation and detection 


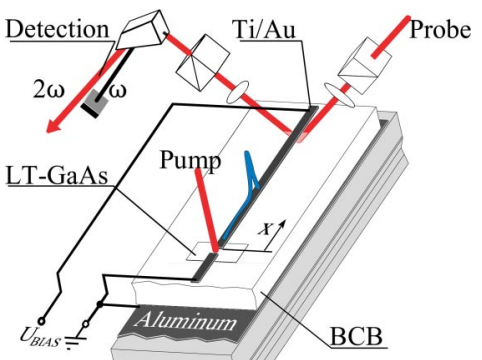

(a)

Silicon
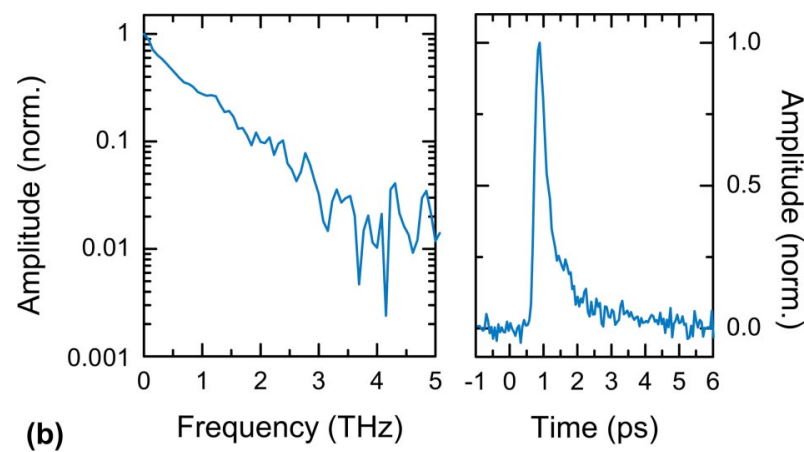

Fig. 8 (online colour at: www.pss-b.com) $\quad$ (a) Experimental setup for time-domain measurement of THz pulses on a TFMSL with an integrated photoconductive switch using EFISH detection in the dielectric of the waveguide. (b) Frequency- and time-domain data of a THz pulse probed at the TFMSL in a distance of $155 \mu \mathrm{m}$ from the PC switch.

[63]. Figure 9(a) shows a single sensor element consisting of a TFMSL-based ring resonator, PC switches (integrated using the method from Fig. 7(a)) and connecting lines. Again, the electric signal is generated at the biased switch, guided along the TFMS lines and through the resonator. The reflected and transmitted signals from the resonator are sampled with the additional PC switches at port 1 and port 2, respectively, by time-delayed optical pulses. The application of integrated PC switches as probes offers the advantages of a fixed measurement configuration, with improved reproducibility. This is achieved, however, at the cost of a reduced detection bandwidth, which is limited by the lifetime of the photogenerated charge carriers. The design of the switches generates only small multi-reflections of the signal that is back-reflected from the resonator. This is very important in order to retrieve undistorted frequency response data from the time-domain signal. Figure 9(b) shows the measured and simulated transmission amplitude data $S_{21}$ in the range of $25-600 \mathrm{GHz}$. The theoretical data are calculated with commercial field-simulation software code (HFSS 9.2.1, Ansoft) based on the finite-element method (FEM). In the model the dielectric constant and the dissipation factor of the polypropylene (PP) layer are assumed to be $\varepsilon_{\mathrm{r}}=2.3$ and $\tan \delta=0.001$, respectively. The measurement data resolve all resonances from the fundamental at $50 \mathrm{GHz}$ up to the tenth harmonic resonance at $500 \mathrm{GHz}$. Thus, there is excellent agreement between measurement and simulation data demonstrating the qualification of this approach for highprecision device characterization.

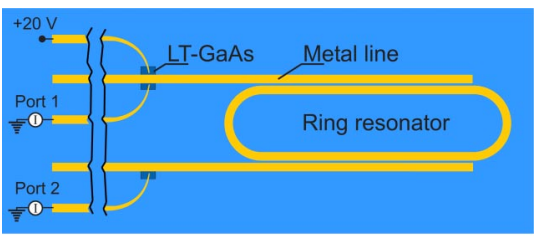

(a)

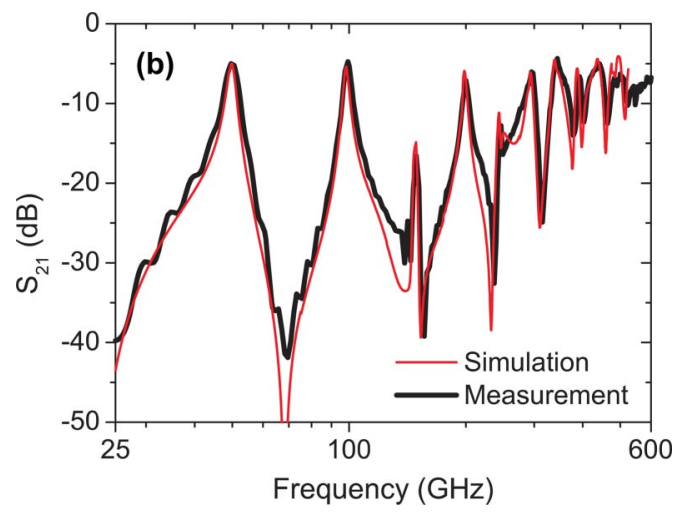

Fig. 9 (online colour at: www.pss-b.com) (a) Scheme of the THz ring resonator device for DNA sensing with integrated PC LT-GaAs switches. (b) Simulated and measured frequency-domain transmission amplitude data of the ring resonator. The $\mathrm{THz}$ signal is generated at the biased PC switch, transmitted through the resonator and detected at port 2. 


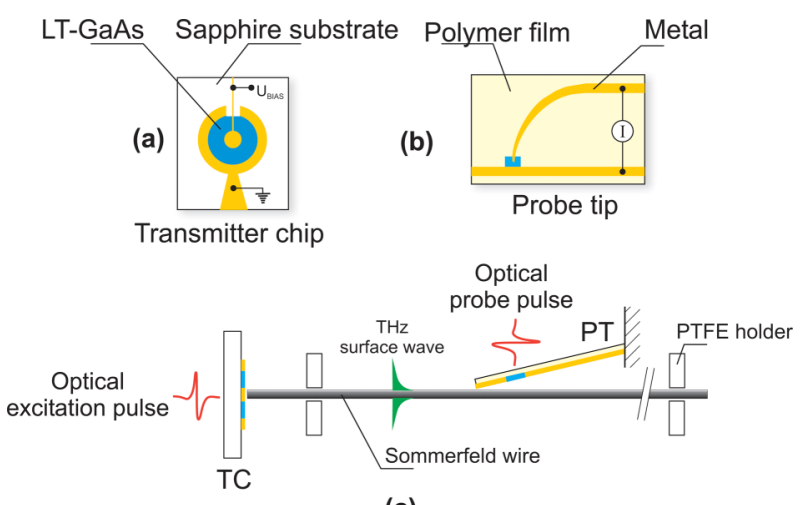

(c)

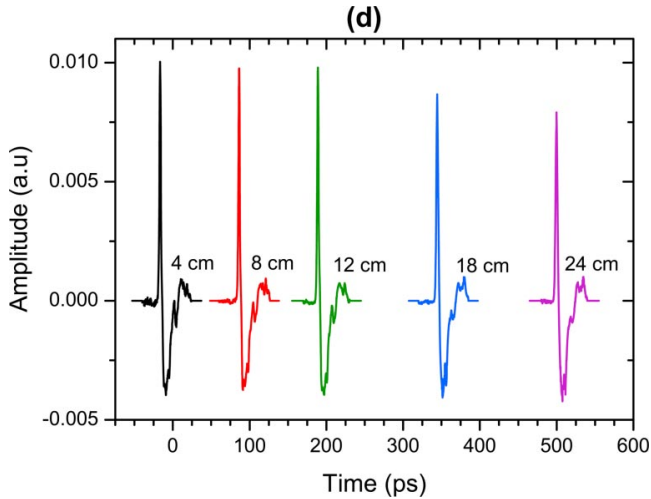

Fig. 10 (online colour at: www.pss-b.com) (a) Schematic plot of the transmitter chip. The inner electrode has a diameter of $90 \mu \mathrm{m}$, the outer electrode has an inner diameter of $290 \mu \mathrm{m}$. (b) Schematic plot of the freely-positionable probe-tip. (c) Experimental setup for propagation constant measurements at a Sommerfeld surface-wave guide. (d) Electric field transients recorded after different transmission distances on a $50 \mu \mathrm{m}$ thick copper-based Sommerfeld wire.

In the following examples the application of bonded LT-GaAs chips is considered for freelypositionable PC probe-tips and antenna devices. As mentioned above, an important advantage of the epitaxial lift-off technique is given by its versatile compatibility with many different host substrates. The PC probe-tips considered here are used to sample electric fields at arbitrary positions at the surface of a device. For this application a flexible probe-tip substrate is highly desirable to reduce mechanical contact forces. Hence, excessive abrasion of the probe and the device under test (DUT) can be avoided. Furthermore, substrates with lowest possible permittivity should be used in order to reduce the dielectric load of the DUT. We have investigated PP foil with a thickness of $35 \mu \mathrm{m}$ for this purpose. It is flexible and has a low permittivity of $\varepsilon_{\mathrm{r}}=2.3$. A metal stripline configuration as sketched in Fig. 10(b) has been structured on the PP substrate after deposition of a LT-GaAs chip. During measurements the signal field is coupled to the continuous straight line, transmitted to the gap at the branch line and probed at the PC switch being in the same place. Since PP is transparent at the wavelength of the optical probe beam $(780 \mathrm{~nm})$ the PC switch can be excited through the substrate, which is essential to enable a small contact angle between the probe-tip and the surface of the DUT. Increasing contact angles will generally lower the coupling efficiency to the probe-tip.

The probe-tip has been applied to characterize the propagation properties of $\mathrm{THz}$ Sommerfeld wire waveguides by recording the amplitude and phase information of transient electric fields after different propagation distances [64]. For this task, a radial LT-GaAs-based PC antenna, as sketched in Fig. 10(a), is used to excite a radially polarized surface-wave (also named as a "Sommerfeld wave") on the singlewire transmission line in the configuration pictured in Fig. 10(c). The time-domain THz field transients probed after propagation distances between $4 \mathrm{~cm}$ and $24 \mathrm{~cm}$ from the transmitter chip are shown in Fig. 10(d). Frequency-domain propagation parameters (not shown here) of the wire have been retrieved from the time-domain data by a procedure described in [55]. Using these freely-positionable PC probetips the frequency-dependent attenuation and dispersion behaviour of Sommerfeld wires was measured in qualitative and quantitative agreement with numerical field simulations [64]. Compared to other approaches, where the field radiated from the end of the waveguide is probed, our method appears to be favorable for waveguide characterization since the field pattern radiated from an open end of a waveguide is highly-frequency dependent [65]. This effect can introduce measurement errors and should be considered especially for waveguides with large cross-section dimensions in the order of the transmitted wavelength.

Yet another application of the epitaxial lift-off technique is in the fabrication of terahertz antenna array structures, as shown exemplary in Fig. 11(a). PC THz emitters are known to suffer from field 


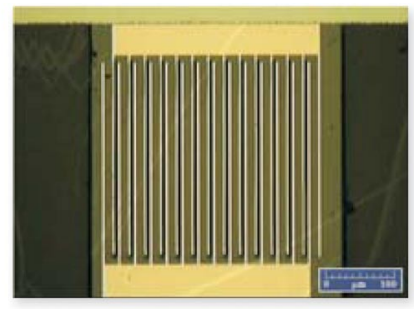

(a)

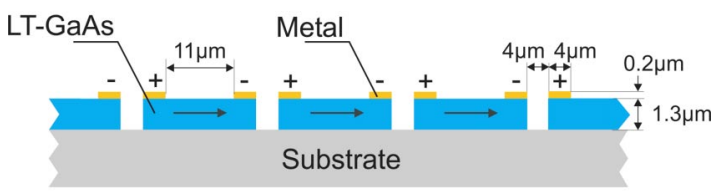

(b)

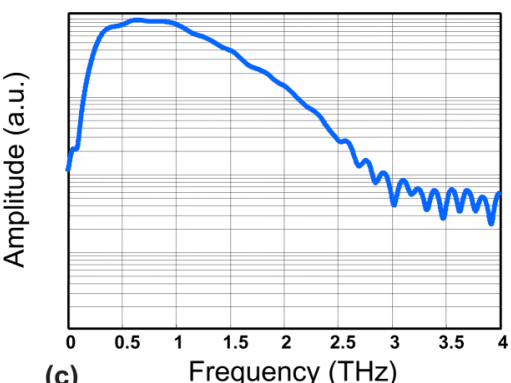

(c)

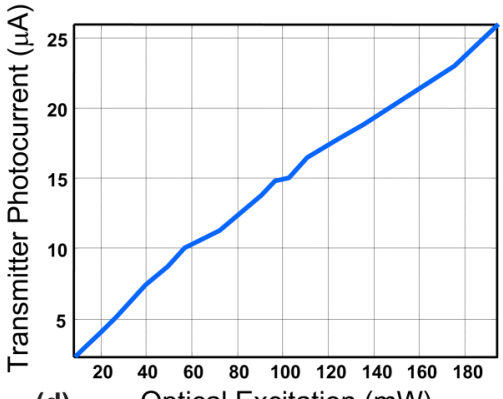

(d)

Fig. 11 (online colour at: www.pss-b.com) (a) Microscope picture of a LT-GaAs antenna array with a lateral size of $290 \mu \mathrm{m} \times 310 \mu \mathrm{m}$, (b) cross-section of the proposed antenna array, (c) measured amplitude spectrum of the antenna array, and (d) measured photo current above averaged optical excitation power showing no effect of saturation.

screening effects caused by the generated charge carriers in the semiconductor material [66]. This effect is hard to avoid and limiting the maximum obtainable THz power of a PC emitter. However, by increasing the optically excited area of the PC emitter the overall $\mathrm{THz}$ power has been increased successfully e.g. with large aperture emitters [67] or antenna arrays [68]. Recently, THz emission was demonstrated with a planar large-area PC emitter consisting of interdigitated electrode metal-semiconductor-metal (MSM) structures on a bulk SI GaAs wafer [69]. This device can be regarded as an array of closely spaced single PC emitters and in this terms it has been investigated theoretically for THz cw-signal generation [70]. The design proposed in Ref. [69] is making use of a layer system consisting of a dielectric and a metallic layer acting as a shadow mask allowing excitation of only every other electrode spacing. Hence, individual array elements interfere constructively in the far-field. In comparison to the largeaperture PC emitters with electrode spacings in the order of $400 \mu \mathrm{m}$, these devices can be driven with bias voltages in the order of only a few tens of volts instead of kilovolts. High voltage sources, however, can cause extreme noise to other measurement devices and should therefore be avoided.

From the cross-sectional view of the antenna array proposed here, in Fig. 11(b), it can be seen that the semiconductor material in between the individual array elements is etched away completely. As important advantages over the former solution the epitaxial lift-off technique outlined in Section 4.1 provides reduced dark current and less processing effort. It yields a self-contained emitter structure on a LT-GaAs semiconductor chip only $1300 \mathrm{~nm}$ thin that can be bonded on a variety of substrates depending on the application requirements. Furthermore, in contrast to SI-GaAs it is also possible to use this chip as a receiver, due to the short carrier lifetimes in LT-GaAs.

Figure 11(c) shows the bandwidth obtainable from an array structure with the dimensions as specified in Fig. 11(a) and (b). With identical optical excitation power of approximately $5 \mathrm{~mW}$, the emitted $\mathrm{THz}$ field using the array structure depicted in Fig. 11(a) is yet 25\% larger compared to a single element emitter [54] in spite of the fact that in this still preliminary configuration only half of the optical excitation power is radiated to the active semiconductor array area. The optical excitation power is distributed over 
a larger area than is the case for a single element, where the optical excitation is tightly focused. This results in an exceedingly smaller excitation density for the array structure and consequently the occurrence of saturation effects not until considerably higher optical power levels. For a single element emitter with a gap size of $10 \mu \mathrm{m}$, saturation typically occurs above $40 \mathrm{~mW}$ of averaged optical excitation power. Fig. 11(d) shows a plot of the transmitter photocurrent drawn at various optical excitations and it can be seen that for optical excitations, where a single element emitter would be well into saturation the antenna array is still in the linear regime of operation. For comparison, the measured $\mathrm{THz}$ field amplitude of this prototype in the present configuration with $150 \mathrm{~mW}$ of optical excitation power is 5.5 fold higher compared to a nearly saturated single element emitter with $40 \mathrm{~mW}$ focused excitation. Further effective optimizations of this principle concept are currently developed and will be presented in the foreseeable future.

\section{Conclusion}

The progress documented in this paper demonstrates clearly the large potentials of III-V semiconductor compunds for future ultrafast photonic and optoelectronic devices and components. Approaching the barriers of silicon dominated mainstream technology, the urgently requested alternatives offered by the coherent and ultrafast III-V phenomena should be reconsidered carefully when designing the concepts "beyond Moore". In a moment where speed and bandwidth become a decisive factor in the ICT-industry, then both pillars of advanced optoelectronics, the coherent phenomena as well as the ultrafast optoelectronic transducers, prepresent valuable and promising starting points.

Acknowledgements We would like to acknowledge the collaboration with numerous members of the Paul-DrudeInstitute, specifically the MBE group providing us with high-quality III-V samples necessary for our work. Especially, we are grateful for discussions with H. T. Grahn, R. Hey and J. Herfort. Above all, the continuous encouragement and support for the joint activities between the Paul-Drude-Institute and our group in Aachen by the director of the Paul-Drude-Institute Klaus Ploog is strongly appreciated.

\section{References}

[1] V. M. Axt and T. Kuhn, Rep. Prog. Phys. 67, 433 (2004).

[2] W. E. Bron, J. Kuhl, and B. K. Rhee, Phys. Rev. B 34, 6961 (1986).

[3] F. Vallée and F. Bogani, Phys. Rev. B 43, 12049 (1991).

[4] F. Vallée, Phys. Rev. B 49, 2460 (1994).

[5] F. Ganikhanov and F. Vallée, Phys. Rev. B 55, 15614 (1997).

[6] C. Thomsen, H. T. Grahn, H. J. Maris, and J. Tauc, Phys. Rev. B 34, 4129 (1986).

[7] H. T. Grahn, H. J. Maris, and J. Tauc, IEEE J. Quantum Electron. 25, 2562 (1989).

[8] T. K. Cheng, J. Vidal, M. J. Zeiger, G. Dresselhaus, M. S. Dresselhaus, and E. P. Ippen, Appl. Phys. Lett. 59, 1923 (1991).

[9] H. J. Zeiger, J. Vidal, T. K. Cheng, E. P. Ippen, G. Dresselhaus, and M. S. Dresselhaus, Phys. Rev. B 45, 768 (1992).

[10] G. C. Cho, W. Kütt, and H. Kurz, Phys. Rev. Lett. 65, 764 (1990).

[11] A. V. Kuznetsov and C. J. Stanton, Phys. Rev. Lett. 73, 3243 (1994).

[12] R. Merlin, Solid State Commun. 102, 207 (1997).

[13] T. Dekorsy, G. C. Cho, and H. Kurz, Light Scattering in Solids, Vol. 8 (Springer Verlag, Berlin, 2000), p. 169.

[14] O. V. Misochko, J. Exp. Theor. Phys. (JETP) 92, 246 (2001).

[15] F. Bloch, Z. Phys. 52, 555 (1928).

[16] C. Zener, Proc. R. Soc. Lond. A 145, 523 (1934).

[17] L. Esaki and R. Tsu, IBM J. Res. Develop. 14, 61 (1970).

[18] E. E. Mendez, F. Agulló-Rueda, and J. M. Hong, Phys. Rev. Lett. 60, 2426 (1988).

[19] P. Voisin, J. Bleuse, C. Bouche, S. Gaillard, C. Alibert, and A. Regreny, Phys. Rev. Lett. 61, 639 (1988).

[20] J. Feldmann, K. Leo, J. Shah, D. A. B. Miller, J. E. Cunningham, T. Meier, G. von Plessen, A. Schulze, P. Thomas, and S. Schmitt-Rink, Phys. Rev. B 46, 7252 (1992). 
[21] K. Leo, P. Haring-Bolivar, F. Brüggemann, R. Schwedler, and K. Köhler, Solid State Commun. 84, 943 (1992).

[22] C. Waschke, H. G. Roskos, R. Schwedler, K. Leo, H. Kurz, and K. Köhler, Phys. Rev. Lett. 70, 3319 (1993).

[23] K. Leo, High Field Transport in Semiconductor Superlattices, Springer Tracts in Modern Physics, Vol. 187 (Springer Verlag, Berlin, 2003).

[24] A. W. Ghosh, L. Jönsson, and J. W. Wilkins, Phys. Rev. Lett. 85, 1084 (2000).

[25] T. Dekorsy, A. Bartels, H. Kurz, K. Köhler, R. Hey, and K. Ploog, Phys. Rev. Lett. 85, 1080 (2000).

[26] A. Mooradian and A. L. McWorther, Phys. Rev. Lett. 19, 849 (1967).

[27] J. Hader, T. Meier, S. W. Koch, F. Rossi, and N. Linder, Phys. Rev. B 55, 13799 (1997).

[28] M. Först, T. Dekorsy, H. Kurz, and R. P. Leavitt, Phys. Rev. B 67, 085305 (2003).

[29] M. Först, T. Dekorsy, H. Kurz, and R. P. Leavitt, phys. stat. sol. (c) 1, 2702 (2004).

[30] B. Rosam, D. Meinhold, F. Löser, V. G. Lyssenko, S. Glutsch, F. Bechstedt, F. Rossi, K. Köhler, and K. Leo, Phys. Rev. Lett. 86, 1307 (2001).

[31] F. W. Smith, A. R. Calawa, C. L. Chen, M. J. Manfra, and L. J. Mahoney, IEEE Electron Device Lett. 9, 77 (1988).

[32] A. Warren, J. M. Woodall, J. L. Freeouf, D. Grischkowsky, D. T. McIntosh, M. R. Melloch, and N. Otsuka, Appl. Phys. Lett. 57, 1331 (1990).

[33] D. C. Look, D. C. Walters, M. O. Manashreh, J. R. Sizelove, C. E. Stutz, and K. R. Evans, Phys. Rev. B 42, $3578(1990)$

[34] S. Gupta, M. Y. Frenkel, J. A. Valdmanis, J. F. Whitaker, G. A. Mourou, F. W. Smith, and A. R. Calawa, Appl. Phys. Lett. 59, 3276 (1991).

[35] X.-Q. Zhou, H. M. van Driel, W. W. Rühle, Z. Gogolak, and K. Ploog, Appl. Phys. Lett. 61, 3020 (1992).

[36] H. M. Heiliger, M. Vossebürger, H. G. Roskos, H. Kurz, R. Hey, and K. Ploog, Appl. Phys. Lett. 69, 2903 (1996).

[37] M. Nagel, T. Meyer, H. M. Heiliger, T. Dekorsy, H. Kurz, R. Hey, and K. Ploog, Appl. Phys. Lett. 72, 1018 (1998).

[38] E. R. Brown, K. A. McIntosh, K. B. Nichols, and C. L. Dennis, Appl. Phys. Lett. 66, 285 (1995).

[39] G. L. Witt, Mater. Sci. Eng. B 22, 9 (1993).

[40] C. Kadow, S. B. Fleischer, J. B. Ibbetson, J. E. Bowers, A. C. Gossard, J. W. Dong, and C. J. Palmstrom, Appl. Phys. Lett. 75, 3548 (1999).

[41] J. Lloyd-Hughes, E. Castro-Camus, M. D. Fraser, C. Jagadish, and M. B. Johnston, Phys. Rev. B 70, 235330 (2004).

[42] G. Segschneider, T. Dekorsy, H. Kurz, R. Hey, and K. Ploog, Appl. Phys. Lett. 71, 2779 (1997).

[43] T. Dekorsy, G. Segschneider, M. Nagel, H. M. Heiliger, R. Hey, K. Ploog, and M. Luysberg, Symposium on Non-Stochiometric III-V Compounds, Erlangen, Germany, October 1998, Vol. 6, p. 85.

[44] T. S. Sosnowski, T. B. Norris, H. H. Wang, P. Grenier, and J. F. Whitaker, Appl. Phys. Lett. 70, 3245 (1997).

[45] G. Segschneider, F. Jacob, T. Löffler, H. G. Roskos, S. Tautz, P. Kiesel, and G. Döhler, Phys. Rev. B 65, 125205 (2002).

[46] D. A. B. Miller, D. S. Chemla, D. J. Eilenberger, P. W. Smith, A. C. Gossard, and W. T. Tsang, Appl. Phys. Lett. 41, 679 (1982).

[47] C. Park, C. G. Park, C. D. Lee, and S. K. Noh, J. Electron. Mater. 26, 1053 (1997).

[48] R. Mendis and D. Grischkowsky, IEEE Microw. Wirel. Compon. Lett. 11, 444 (2001).

[49] M. Nagel, P. H. Bolivar, and H. Kurz, Semicond. Sci. Technol. 20, 281 (2005).

[50] L. J. Chen, H. W. Chen, T. F. Kao, J. Y. Lu, and C. K. Sun, Opt. Lett. 31, 308 (2006).

[51] M. Nagel, A. Marchewka, and H. Kurz, Opt. Express 14, 9944 (2006).

[52] E. Yablonovitch, D. M. Hwang, T. J. Gmitter, L. T. Florez, and J. P. Harbison, Appl. Phys. Lett. 56, 2419 (1990).

[53] L. Desplanque, J. F. Lampin, and F. Mollot, Appl. Phys. Lett. 84, 2049 (2004).

[54] M. Awad, M. Nagel, H. Kurz, J. Herfort, and K. Ploog, Appl. Phys. Lett. (in preparation)

[55] H.-M. Heiliger, M. Nagel, H. G. Roskos, H. Kurz, F. Schnieder, W. Heinrich, R. Hey, and K. Ploog, Appl. Phys. Lett. 70, 2233 (1997).

[56] M. Nagel, P. H. Bolivar, M. Brucherseifer, H. Kurz, A. Bosserhoff, and R. Büttner, Appl. Phys. Lett. 80, 154 (2002).

[57] T. Ohkubo, M. Onuma, J. Kitagawa, and Y. Kadoya, Appl. Phys. Lett. 88, 212511 (2006). 
[58] J. Cunningham, C. Wood, A. G. Davies, C. K. Tiang, P. Tosch, D. A. Evans, E. H. Linfield, I. C. Hunter, and M. Missous, Appl. Phys. Lett. 88, 071112 (2006).

[59] T. Ouchi, S. Kasai, R. Kurosaka, T. Itsuji, H. Yoneyama, M. Yamashita, K. Kawase, and H. Ito, Conference Digest of the IRMMW/THz2006, Shanghai, China, 2006, p. 273.

[60] C. Wood, J. Cunningham, P. C. Upadhya, E. H. Linfield, I. C. Hunter, A. G. Davies, M. Missous, Appl. Phys. Lett. 88, 142103 (2006).

[61] M. Nagel, C. Meyer, H.-M. Heiliger, T. Dekorsy, H. Kurz, R. Hey, and K. Ploog, Appl. Phys. Lett. 72, 1018 (1998).

[62] T. Pfeifer, H.-M. Heiliger, T. Löffler, C. Ohlhoff, C. Meyer, G. Lüpke, H. G. Roskos, and H. Kurz, IEEE J. Sel. Top. Quantum Electron. 2, 586 (1996).

[63] M. Nagel, F. Richter, P. Haring Bolivar, and H. Kurz, Phys. Med. Biol. 48, 3625-3636 (2003).

[64] M. Wächter, M. Nagel, and H. Kurz, Opt. Express 13, 10815-10822 (2005).

[65] J. A. Deibel, N. Berndsen, K. Wang, D. M. Mittleman, N. C. van der Valk, and P. C. M. Planken, Opt. Express 14, 8772-8778 (2006).

[66] M. Tani, S. Matsuura, K. Sakai, and S. Nakashima, Appl. Opt. 36, $7853-7859$ (1997).

[67] G. Zhao, R. N. Schouten, N. van der Valk, W. T. Wenckebach, and P. C. M. Planken, Rev. Sci. Instrum. 73, $1715-1719$ (2002).

[68] H. Yoneda, K. Tokuyama, K. Ueda, H. Yamamoto, and K. Baba, Appl. Opt. 40, 6733-6736 (2001).

[69] A. Dreyhaupt, S. Winnerl, T. Dekorsy, and M. Helm, Appl. Phys. Lett. 86, 121114 (2005).

[70] D. Saeedkia, R. R. Mansour, and S. Safavi-Naeini, IEEE Trans. Antennas Propag. 53, 4044-4050 (2005). 